\title{
Evodiamine inhibits PDGF-BB-induced proliferation of rat vascular smooth muscle cells through the suppression of cell cycle progression and oxidative stress
}

\author{
XIE GE, SI-YU CHEN, MEI LIU, TING-MING LIANG and CHANG LIU \\ Jiangsu Key Laboratory for Molecular and Medical Biotechnology and College of Life Sciences, \\ Nanjing Normal University, Nanjing, Jiangsu 210023, P.R. China
}

Received September 12,2015; Accepted September 19, 2016

DOI: $10.3892 / \mathrm{mmr} .2016 .5798$

\begin{abstract}
Vascular smooth muscle cell (VSMC) proliferation is a key event in the development of in-stent restenosis. Evodiamine is an indole alkaloid extracted from the Chinese medicine, evodia, and has been shown to inhibit tumor cell proliferation and protect the cardiovascular system. However, whether evodiamine affects VSMC proliferation remains to be elucidated. Therefore, the present study examined the effects and the mechanisms of action of evodiamine on the proliferation of rat VSMCs. The cells were treated with evodiamine alone or in combination with platelet-derived growth factor-BB (PDGF-BB) stimulation. It was found that evodiamine inhibited PDGF-BB-induced VSMC proliferation in a dose-dependent manner, without inducing cell death. Evodiamine also retarded cell cycle progression, evidenced by the suppression of the expression of cell cycle-promoting cyclin proteins and cyclin-dependent kinases. In addition, evodiamine attenuated the PDGF-BB-induced phosphorylation of mitogen-activated protein kinases p38 and extracellular signal-regulated kinases $1 / 2$, however, it had no effect on the phosphorylation of Akt. Evodiamine also inhibited the increase of reactive oxygen species generation and upregulated the mRNA expression levels of genes encoding antioxidant enzymes. These findings provide important insights into the mechanisms underlying the vasoprotective actions of evodiamine and suggest that it may be a useful therapeutic agent for the treatment of vascular occlusive disease.
\end{abstract}

Correspondence to: Dr Chang Liu, Jiangsu Key Laboratory for Molecular and Medical Biotechnology and College of Life Sciences, Nanjing Normal University, 1 Wenyuan Road, Nanjing, Jiangsu 210023, P.R. China

E-mail: changliu@njnu.edu.cn

Key words: evodiamine, vascular smooth muscle cells, proliferation, cell cycle, oxidative stress

\section{Introduction}

The aberrant and accelerated proliferation of vascular smooth muscle cells (VSMCs) is the major biological process underlying certain pathological conditions, including in-stent restenosis, the pathological re-narrowing of the vessel lumen following surgical intervention for vascular stenosis (1). Such vascular lesions are typically multifactorial and are most often dependent on the release of growth factors, including platelet-derived growth factor-BB (PDGF-BB). The expression of PDGF-BB is known to be increased following vascular injury, which further activates cell proliferation signaling by binding to PDGF receptor $\beta$ (2). Under physiological conditions, VSMCs remain in a quiescent state and express $\alpha$-smooth muscle actin, desmin and smoothelin. However, in response to various mitogenic stimuli, including PDGF-BB, VSMCs may switch to a state of high proliferation, resulting in decreased expression levels of these markers (3). Furthermore, cell cycle progression and the expression levels of cell cycle-associated proteins have been found to be upregulated by PDGF-BB in VSMCs (4). To overcome VSMC proliferation-mediated restenosis, drug-eluting stents have been developed, aimed at inhibiting VSMC growth through the release of antiproliferative substances, including paclitaxel and rapamycin (5-7). However, unresolved problems with these compounds include impaired re-endothelialization and the subsequent induction of thrombosis (8), which makes the characterization of other compounds with the ability to suppress VSMC proliferation of clinical relevance. In accordance, the implication of natural plant-derived compounds in controlling the proliferation of VSMCs in diseased arteries has been widely investigated in the last decade.

The fruit of 'Wu-Zhu-Yu' (Evodiae fructus) of Evodia rutaecarpa Benth (Rutaceae) is one of the most popular and multi-purpose herbs traditionally used in China for the treatment of headaches, abdominal pain, menstrual problems, vomiting, diarrhea and other diseases (9). Phytochemical studies have shown the presence of evodiamine (Fig. 1A), which is an indole alkaloid present in high levels in the Chinese medicine, evodia. Evodiamine has a wide variety of bioactivities with antinociceptive, anti-obesity, vasodilatory, antitumor and anti-inflammatory effects (10). Of note, evodiamine 
exhibits antitumor properties by inhibiting the proliferation of various cancer cell lines. The molecular mechanisms through which evodiamine suppresses proliferation rates involve cell cycle progression arrest (G2/M phase) and the induction of apoptosis (11). Of note, evodiamine has a beneficial effect in cardiovascular diseases. For example, evodiamine causes vasodilation in mesenteric arteries isolated from rats and its effect is endothelium-dependent (12). Evodiamine also has a significant diuretic effect due to the inhibition of aldosterone release, which can control blood volume (13). In addition, evodiamine inhibits light-induced production of reactive oxygen species (ROS) and pro-inflammatory cytokines, phosphorylation of mitogen-activated protein kinases (MAPKs) p38 and extracellular signal-regulated kinases 1/2 (Erk1/2), and activation of NADPH oxidase in human monocytes (14). These findings suggest that evodiamine has the potential to treat cardiovascular diseases.

Although evodiamine has been demonstrated to inhibit the proliferation of tumor cells and is beneficial for the cardiovascular system, whether evodiamine regulates the pathophysiological processes of VSMCs remains to be elucidated. Therefore, the aim of the present study was to investigate the antiproliferative activity and the mechanistic target of evodiamine in PDGF-BB-stimulated VSMCs. The findings provided evidence that evodiamine suppressed VSMC proliferation and cell cycle progression via regulating the expression of cell cycle-associated proteins and the activation of MAPKs p38 and Erk1/2, and inhibiting the production of ROS.

\section{Materials and methods}

Materials. Evodiamine was purchased from Selleck Chemicals (Houston, TX, USA), and dissolved in DMSO to a $2 \mathrm{mmol} / \mathrm{l}$ stock solution for later use. PDGF-BB was purchased from Sigma-Aldrich; Merck Millipore (Darmstadt, Germany) and dissolved in $4 \mathrm{mmol} / 1$ hydrochloric acid containing $0.1 \%$ bovine serum albumin.

Cell culture. The rat VSMCs were isolated using an explant technique, as previously described (15). In brief, the thoracic aortas were isolated from three male Sprague Dawley rats sacrificed by cervical dislocation at the age of 3-4 weeks (provided by the Laboratory Animal Center at Nanjing Normal University, Nanjing, China). The rats were housed on a 12/12 h light/dark cycle at $18-26^{\circ} \mathrm{C}$ and had free access to food and water. The middle vascular layers comprising the major localization of VSMCs were carefully dissected and cut into small sections for explant. The VSMCs were cultured in $5 \% \mathrm{CO}_{2}$ at $37^{\circ} \mathrm{C}$ using Dulbecco's modified Eagle's medium (DMEM; Gibco; Thermo Fisher Scientific, Inc., Waltham, MA, USA) supplemented with $10 \%$ fetal bovine serum (FBS; Gibco; Thermo Fisher Scientific, Inc.). The cells at passages 4-8 were used in all experiments. The study was approved by the Laboratory Animal Welfare and Ethics Committee of Nanjing Normal University (Nanjing, China).

Cell viability assay. To analyze VSMC viability, a CCK-8 toxicity assay was used. Briefly, $5 \times 10^{3}$ VSMCs were seeded into each well of 96-well plates, cultured at $37^{\circ} \mathrm{C}$ overnight for attachment, and treated with evodiamine $(0.1,0.5,1,2$ or
$4 \mu \mathrm{M})$ in $100 \mu \mathrm{l}$ medium for $30 \mathrm{~h}$. Following treatment, $10 \mu \mathrm{l}$ WST-8 reagent (EnoGene, Nanjing, China) was added to each well and incubated at $37^{\circ} \mathrm{C}$ for $2 \mathrm{~h}$. Finally, a microplate reader was used to measure the absorbance at $450 \mathrm{~nm}$.

Cell proliferation assay. To analyze VSMC proliferation, a CCK-8 proliferation assay, direct cell counting and an EdU incorporation assay were used. For the CCK-8 assay, $2 \times 10^{3}$ VSMCs were seeded into each well of 96 -well plates and incubated at $37^{\circ} \mathrm{C}$ overnight for attachment. Subsequently, the cells were pre incubated with $0,0.1$ or $0.5 \mu \mathrm{M}$ evodiamine for $6 \mathrm{~h}$, and then challenged with $10 \mathrm{ng} / \mathrm{ml}$ PDGF-BB for $24 \mathrm{~h}$ in the presence or absence of evodiamine as treated in the pre-incubation. The cells were incubated in $5 \% \mathrm{CO}_{2}$ at $37^{\circ} \mathrm{C}$ using DMEM without FBS supplementation throughout this experiment. Following treatment of the cells, WST-8 reagent was added and processed, as described above.

For the direct cell counting, $5 \times 10^{4}$ VSMCs were seeded into each well of 6-well plates. Following a similar treatment procedure to that described above, the cells were resuspended with $0.05 \%$ trypsin and $0.02 \%$ EDTA, and counted using a hemocytometer.

An EdU incorporation assay was used to analyze cell proliferation through measuring DNA synthesis. In brief, $50 \mu \mathrm{M}$ EdU (Guangzhou RiboBio Co., Ltd., Guangzhou, China) was added to the medium for $2 \mathrm{~h}$ following treatment of the cells, and the cells were then fixed with $4 \%$ paraformaldehyde. EdU incorporation was determined by incubating the cells with 1X Apollo ${ }^{\circledR} 567$ reaction reagent (cat. no. C10310-1; Guangzhou RiboBio Co., Ltd.) at room temperature for $30 \mathrm{~min}$ in the dark. The cells were counterstained with DAPI (Sigma-Aldrich; Merck Millipore). Using a fluorescence microscope, EdU-positive (pink) cells and DAPI-stained nuclei (blue) were counted, respectively, and the average ratios between the were calculated for statistical analysis.

Flow cytometry. Following treatment similar to that described for the cell proliferation assay, the VSMCs were fixed in $70 \%$ ethanol at $-20^{\circ} \mathrm{C}$ overnight, washed once with PBS and incubated with PBS containing RNase A (100 $\mu \mathrm{g} / \mathrm{ml}$; Vazyme Biotech, Nanjing, China) at $37^{\circ} \mathrm{C}$ for $30 \mathrm{~min}$. The cells were then stained with prodium iodide (Vazyme Biotech) at $4^{\circ} \mathrm{C}$ for $1 \mathrm{~h}$. Fluorescence was measured and analyzed using a FACSCalibur flow cytometer (BD Biosciences, San Jose, CA, USA). The distributions of cells at the $\mathrm{G}_{0} / \mathrm{G}_{1}, \mathrm{~S}$ and $\mathrm{G}_{2} / \mathrm{M}$ phases were determined using Modfit LT software (version 3.1; BD Biosciences).

Reverse transcription-quantitative polymerase chain reaction (RT-qPCR) analysis. Total RNA $(1 \mu \mathrm{g})$ was mixed with $2 \mu \mathrm{l}$ 5X qRT SuperMix from the HiScript ${ }^{\mathrm{TM}}$ Q RT SuperMix kit for qPCR (cat. no. R122-01; Vazyme Biotech), RNase free water was added to make a total volume of $10 \mu \mathrm{l}$, and then RT was performed at $50^{\circ} \mathrm{C}$ for $15 \mathrm{~min}$ to produce cDNA. The mRNA levels of heme oxygenase 1 (HO-1), glutathione peroxidase 1 (GPx-1), superoxide dismutase (SOD) 1 and SOD2 were quantified by RT-qPCR using AceQ qPCR SYBR Green Master Mix (cat. no. Q111; Vazyme Biotech). In detail, the cDNAs acquired after RT were diluted 10 times, then $2 \mu \mathrm{l}$ was mixed with $5 \mu$ l AceQ qPCR SYBR Green Master Mix 
and $50 \mu \mathrm{M}$ primers $(0.15 \mu \mathrm{l}$ each $)$, and distilled deionized water was added to make a total volume of $10 \mu \mathrm{l}$. The samples were amplified using the LightCycler ${ }^{\circledR}$ Nano system (Roche Diagnostics, Basel, Switzerland). The amplification conditions were: $95^{\circ} \mathrm{C}$ for $10 \mathrm{~min}$ for initial denaturation, and 45 cycles of amplification consisting of $95^{\circ} \mathrm{C}$ for $10 \mathrm{sec}$, and $60^{\circ} \mathrm{C}$ for $30 \mathrm{sec}$. $18 \mathrm{~S}$ ribosomal RNA served as an internal control to normalize the expression levels of mRNAs. The quantification cycle $(\mathrm{Cq})$ values were calculated using the instrument software, and the relative expression levels were calculated using the $2^{-\Delta \Delta C \mathrm{q}}$ method (16). The primer sequences were as follows: HO-1, forward 5'-TTTCACCTTCCCGAGCAT-3' and reverse: 5'-GCCTCTTCTGTCACCCTGT-3'; GPX-1, forward 5'-ACATCAGGAGAATGGCAAGA-3' and reverse 5'-CCG CAGGAAGGTAAAGAGC-3'; SOD1, forward 5'-GGTCCA CGAGAAACAAGA-3' and reverse 5'-AGACTCAGACCA CATAGGGA-3'; SOD2, forward 5'-GCAAGGTCGCTTACA GAT-3' and reverse 5'-ATGGCTTTCAGATAGTCAGGTC-3'; $18 \mathrm{~S}$, forward 5'-AAACGGCTACCACATCCAAG-3' and reverse 5'-CCTCCAATGGATCCTCGTTA-3'.

Western blot analysis. The VSMCs were lysed in RIPA buffer containing $50 \mathrm{mmol} / \mathrm{l}$ Tris- $\mathrm{HCl}$ (pH 8.0), $150 \mathrm{mmol} / \mathrm{l}$ $\mathrm{NaCl}, 1 \%$ NP-40, $1 \%$ sodium deoxycholate, $0.1 \%$ SDS, $0.1 \mathrm{mmol} / 1 \mathrm{DTT}, 0.002 \mathrm{mg} / \mathrm{ml}$ leupeptin, $1 \mathrm{mmol} / 1 \mathrm{NaVO}_{3}$, $0.05 \mathrm{mmol} / 1 \mathrm{PMSF}$ and $0.002 \mathrm{mg} / \mathrm{ml}$ aprotinin. The protein concentrations were quantified using Dc protein assay reagent (Bio-Rad Laboratories, Inc., Hercules, CA, USA), and $20 \mu \mathrm{g}$ proteins were loaded and separated using 10\% SDS-PAGE. The proteins were then transferred onto PVDF membranes (EMD Millipore, Bedford, MA, USA). After blocking with $5 \%$ non-fat milk (blocking buffer) at room temperature for $1 \mathrm{~h}$, the membranes were incubated overnight at $4^{\circ} \mathrm{C}$ with appropriate primary antibodies diluted in blocking buffer as follows: Rabbit monoclonal cyclin-dependent kinase (CDK)2 (1:500; cat. no. 2546; Cell Signaling Technology, Inc., Danvers, MA, USA), mouse monoclonal CDK4 (1:500; cat. no. 610147; BD Biosciences), mouse monoclonal CDK6 (1:500; cat. no. 3136; Cell Signaling Technology, Inc.), mouse monoclonal p21 (1:500; cat. no. 556430; BD Biosciences), rabbit monoclonal p27 (1:500; cat. no. 3,686; Cell Signaling Technology, Inc.), rabbit monoclonal cyclin D1 (1:500; cat. no. 2978; Cell Signaling Technology, Inc.), mouse monoclonal cyclin E (1:500; cat. no. 4129; Cell Signaling Technology, Inc.), mouse monoclonal proliferating cell nuclear antigen (PCNA; 1:500; cat. no. 2586; Cell Signaling Technology, Inc.), mouse monoclonal phospho-p38 (Thr180/Try182; 1:500; cat. no. 9216; Cell Signaling Technology, Inc.), rabbit polyclonal total p38 MAPK (1:500; cat. no. 9212; Cell Signaling Technology, Inc.), rabbit monoclonal phospho-Erk1/2 (1:500; cat. no. 4377; Cell Signaling Technology, Inc.), rabbit polyclonal total Erk1/2 (1:500; cat. no. 9102; Cell Signaling Technology, Inc.), rabbit polyclonal phospho-Akt (Ser473; 1:500; cat. no. 9271; Cell Signaling Technology, Inc.), rabbit polyclonal total Akt (1:500; cat. no. 9272; Cell Signaling Technology, Inc.) and mouse monoclonal GAPDH (1:5,000; cat. no. KC-5G5; KangChen Biotech, Inc., Shanghai, China). Following washing with PBS with $0.1 \%$ Tween-20 (PBST) three times, the membranes were incubated at room temperature for $1 \mathrm{~h}$ with anti-mouse (1:2,000; cat. no. sc-2005) or anti-rabbit $(1: 2,000$; cat. no. sc-2004) horseradish peroxidase conjugated secondary antibodies (Santa Cruz Biotechnology, Inc., Dallas, TX, USA). Subsequently, the membranes were washed with PBST three times, and the bands of target proteins were visualized using Pierce $^{\mathrm{TM}}$ ECL Western Blotting Substrate (Thermo Fisher Scientific, Inc.) and Tanon-5200 Chemiluminescent Imaging System (Tanon Science \& Technology, Ltd., Shanghai, China). The relative intensity of the target bands were quantified by densitometric scanning using Image $\mathrm{J} 1.32 \mathrm{j}$ software (National Institutes of Health, Bethesda, MD, USA).

Measurement of ROS generation. To measure ROS generation in the VSMVs, 2', 7' dichlorofluorescin diacetate (DCFH-DA) was used. Briefly, following pretreatment with $0,0.1$ or $0.5 \mu \mathrm{M}$ evodiamine for $24 \mathrm{~h}$, the VSMCs were stimulated with $10 \mathrm{ng} / \mathrm{ml}$ PDGF-BB for $1 \mathrm{~h}$, and then loaded with $10 \mu \mathrm{M}$ DCFH-DA for $1 \mathrm{~h}$ (Beyotime Institute of Biotechnology, Inc., Nantong, China). These treatments were performed at $37^{\circ} \mathrm{C}$. The VSMCs were then rinsed twice with PBS, and images were captured with a fluorescence microscope.

Statistical analysis. Graphpad Prism 5 software (GraphPad Software, Inc., San Diego, CA, USA) was used to analyze the data in the present study. Groups of data are presented as the mean + standard deviation. To compare the data between two groups, Student's unpaired $t$-test was used. $\mathrm{P}<0.05$ was considered to indicate a statistically significant difference.

\section{Results}

Evodiamine inhibits PDGF-BB-induced VSMC proliferation. Safety is of highest priority in drug development. Therefore, the present study first evaluated the possible cell toxicity induced by evodiamine using a CCK-8 assay. As shown in Fig. 1B, treating the VSMCs with evodiamine alone at concentrations $<1 \mu \mathrm{M}$ for $30 \mathrm{~h}$ did not affect the cell viability. Therefore, doses between 0.1 and $0.5 \mu \mathrm{M}$ were considered safe for VSMCs, and were used throughout the present study. The effect of evodiamine on VSMC proliferation was then assessed. To ensure maximum accuracy, three methods were used to quantify the cell proliferation rate. Although the detailed data differed, the results generated from these methods shared similar trends. In general, it was found that evodiamine treatment did not affect the basal level of VSMC proliferation. By contrast, PDGF-BB significantly accelerated cell growth by 1.79-4.91 fold, compared with the control, according to the different methods. Furthermore, evodiamine inhibited PDGF-BB-induced VSMC proliferation in a dose-dependent manner. For the CCK-8 assay, the rates of inhibition were 19.65 and $26.77 \%$ when the doses of evodiamine were 0.1 and $0.5 \mu \mathrm{M}$, respectively (Fig. 1C). For the cell counting assay, the rates of inhibition were 31.15 and $47.54 \%$ (Fig. 1D), and for the EdU incorporation assay, they were 31.84 and $47.13 \%$, with pink dots representing EdU-incorporated nuclei in Fig. 1E and F.

Evodiamine inhibits cell cycle progression. As cell cycle progression is tightly associated with accelerated cellular proliferation, the present study used flow cytometry to investigate how evodiamine affects the cell cycle phases. As shown in Fig. 2A and B, PDGF-BB significantly increased 
<smiles>CN1c2ccccc2C(=O)N2CCc3c([nH]c4ccccc34)C21</smiles>

B

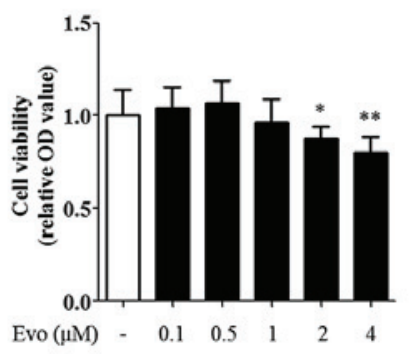

C

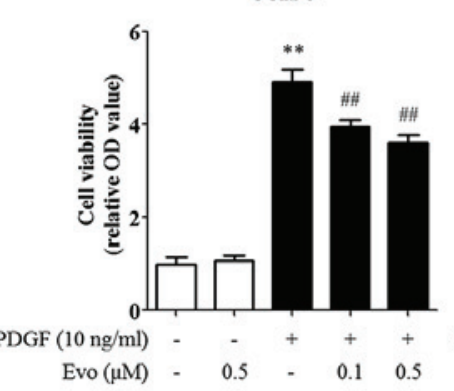

D

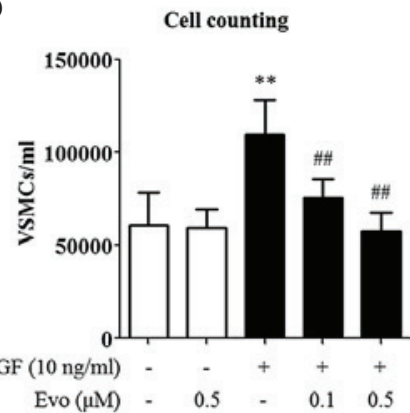

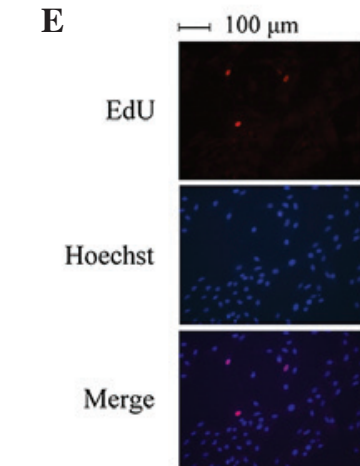

PDGF $(10 \mathrm{ng} / \mathrm{ml})$

Evo $(\mu \mathrm{M})$

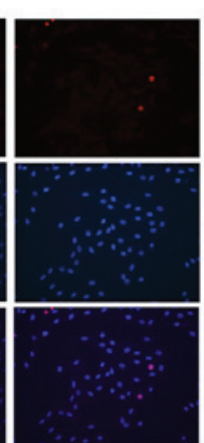

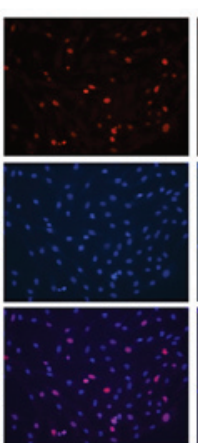

$+$

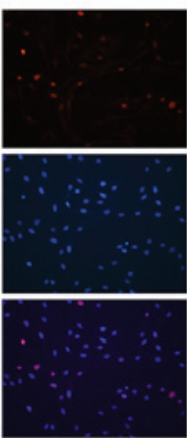

$+$

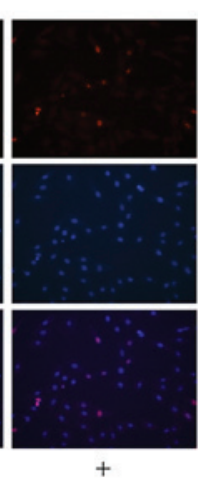

0.5

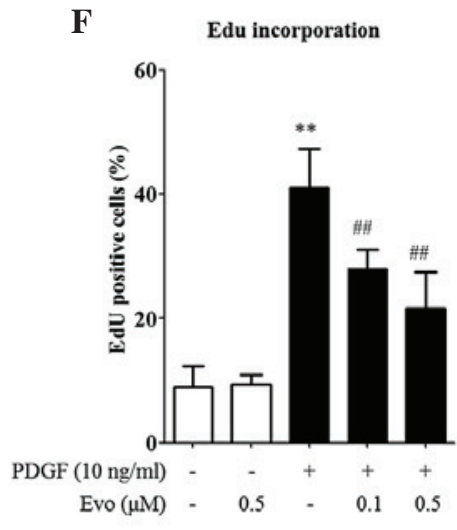

Figure 1. Evodiamine inhibits PDGF-BB-induced VSMC proliferation. (A) Chemical structure of evodiamine. To measure cell toxicity, (B) VSMCs were treated with $0.1,0.5,1,2$ or $4 \mu \mathrm{M}$ evodiamine for $30 \mathrm{~h}$, followed by a CCK- 8 analysis. To measure cell proliferation, VSMCs were pretreated with 0.1 or $0.5 \mu \mathrm{M}$ evodiamine for $6 \mathrm{~h}$ and then stimulated with $10 \mathrm{ng} / \mathrm{ml}$ PDGF-BB for $24 \mathrm{~h}$. Cell proliferation was determined using a (C) CCK-8 assay, (D) direct cell counting and an (E and F) EdU incorporation assay. Magnification, $x 200$. Data are presented as the mean + standard deviation of three independently prepared samples, each with six measurements. $\mathrm{P}<0.05$ and ${ }^{* *} \mathrm{P}<0.01$, compared with the control group; ${ }^{\# \#} \mathrm{P}<0.01$, compared with the PDGF-BB-stimulated group. Evo, evodiamine; PDGF-BB, platelet-derived growth factor-BB; VSMCs, vascular smooth muscle cells; OD, optical density.

the percentage of cells in the S-phase, from 0.46 to $4.58 \%$, and $\mathrm{G}_{2} / \mathrm{M}$ phase, from 0.49 to $5.51 \%$. Correspondingly, the percentage of cells at the $\mathrm{G}_{0} / \mathrm{G}_{1}$ phase were reduced from 99.04 to $89.91 \%$. By contrast, the administration of evodiamine antagonized the effects of PDGF-BB and caused an increase in the percentage of cells arrested in the $\mathrm{G}_{0} / \mathrm{G}_{1}$ phase $(94.67 \%$ of the total cells). These data suggested that the inhibition of VSMC proliferation by evodiamine may be due to cell cycle arrest.

Evodiamine regulates cell cycle-associated proteins. The cell cycle is finely controlled by a series of regulatory proteins, therefore, the present study assessed changes in the expression of these proteins caused by evodiamine. As shown in Fig. 3A and B, evodiamine reduced the protein expression level of PCNA, confirming its inhibitory effects on VSMC proliferation. By contrast, cyclins and CDKs orchestrate cell cycle progression. It was found that the PDGF-BB-induced protein expression levels of CDK2/4/6 and cyclin D1/E, were suppressed by evodiamine in a dose-dependent manner (Figs. 3C-G). p21 and p27 are negative regulators of cyclin-CDK complexes and the present study found that the protein expression of p21 was inhibited by evodiamine (Fig. 3A and $\mathrm{H}$ ), whereas that of p27 was induced by evodiamine (Fig. 3A and I).

Evodiamine inhibits PDGF-BB-induced kinase activation. MAPKs, including p38 and Erk1/2, and serine/threonine kinase Akt are involved in the regulation of PDGF-BB-induced
VSMC proliferation. As shown in Fig. 4A-D, the levels of phosphorylated (active) p38, Erk1/2 and Akt were all increased by PDGF-BB in the VSMCs, as expected. However, the administration of evodiamine markedly decreased the phosphorylation of p38 and Erk1/2, particularly p38, whereas the effect on Akt phosphorylation was more modest, indicating the regulation specificity of evodiamine in kinase activation.

Evodiamine inhibits PDGF-BB-induced ROS generation in $V S M C s$. ROS-induced oxidative stress is important in the initiation of VSMC dysfunction. To directly evaluate the effect of evodiamine on ROS production, the present study quantified ROS levels in the PDGF-BB-treated VSMCs. As shown in Fig. 5A and B, treatment with evodiamine alone did not alter the basal level of ROS generation, whereas treatment with PDGF-BB $(10 \mathrm{ng} / \mathrm{ml})$ for $1 \mathrm{~h}$ caused a marked increase (8.78-fold) in DCFH-DA fluorescence, compared with the control cells. However, pretreatment with evodiamine for $24 \mathrm{~h}$ significantly inhibited ROS generation. To investigate the possibility that evodiamine attenuated oxidative stress via the induction of antioxidant enzymes, the present study analyzed the mRNA expression levels of genes encoding key antioxidant enzymes, including HO-1, GPx-1, SOD1 and SOD2. It was found that PDGF-BB stimulation inhibited the mRNA expression levels of HO-1 and GPx-1, but increased those of SOD2. Evodiamine increased the basal expression levels of HO-1, GPx-1 and SOD2. Of note, evodiamine induced all four antioxidant genes examined in 

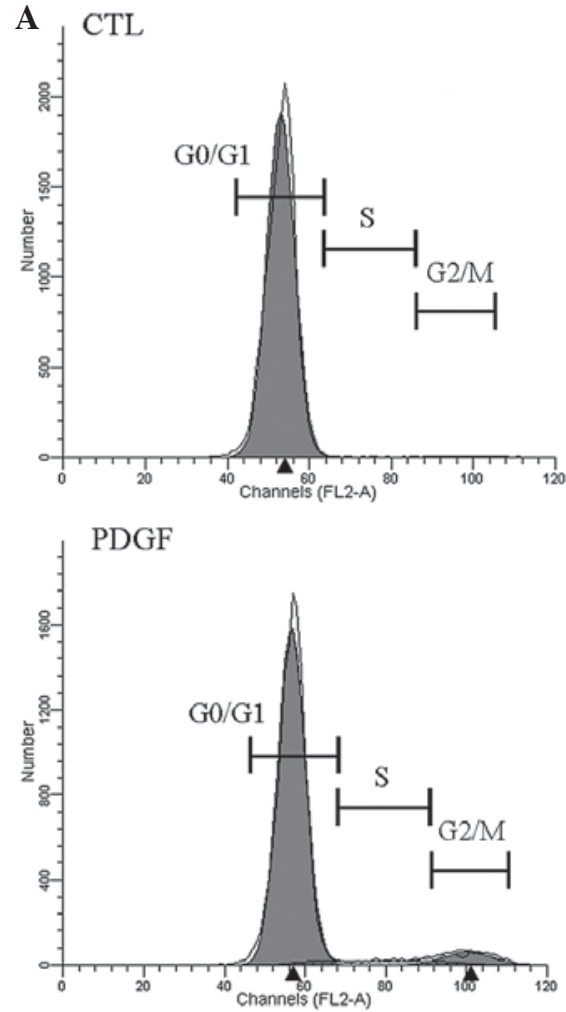
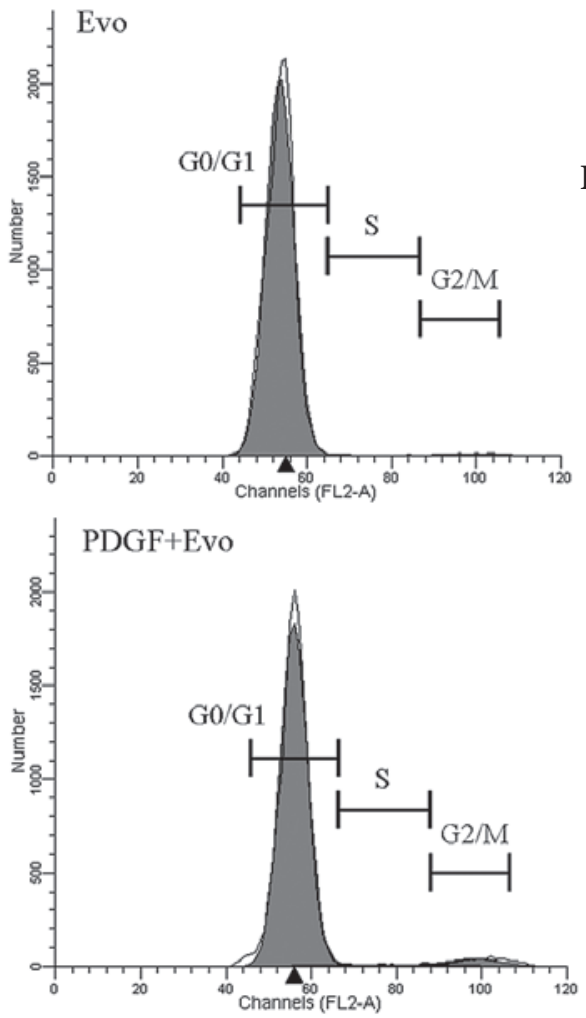

B

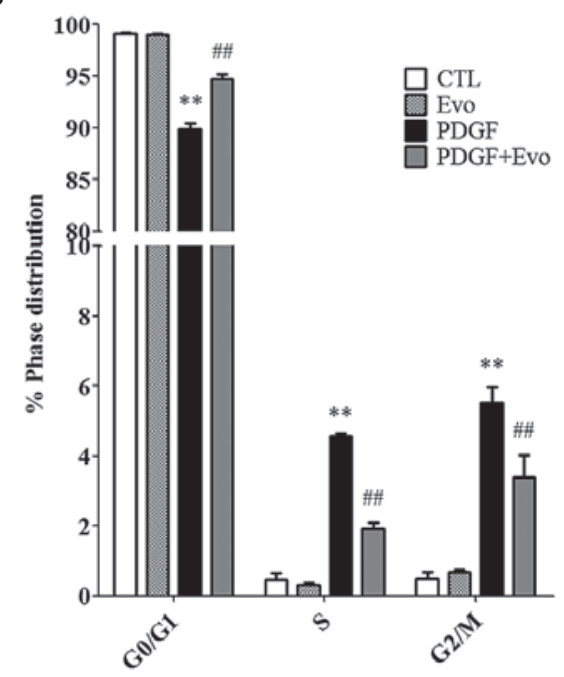

Figure 2. Evodiamine inhibits cell cycle progression. Vascular smooth muscle cells were pretreated with $0.5 \mu \mathrm{M}$ evodiamine for $6 \mathrm{~h}$ and then stimulated with $10 \mathrm{ng} / \mathrm{ml}$ PDGF-BB for $24 \mathrm{~h}$. (A) Cell cycle phase distributions were assessed using flow cytometry. (B) Results of the statistical analysis. Data are presented as the mean + standard deviation of three independently prepared samples, each with five measurements. ${ }^{* *} \mathrm{P}<0.01$, compared with the control group; ${ }^{\# \#} \mathrm{P}<0.01$, compared with the PDGF-BB-stimulated group. Evo, evodiamine; PDGF-BB, platelet-derived growth factor-BB.

a concentration-dependent manner in the PDGF-BB-treated VSMCs (Fig. 5C-F).

\section{Discussion}

In the present study, it was demonstrated that evodiamine inhibited PDGF-BB-induced VSMC proliferation in a dose-dependent manner without causing cell toxicity. Treatment with evodiamine inhibited progression of the cell cycle, suppressed activation of the p38 and Erk MAPK pathways and ameliorated the generation of ROS. To the best of our knowledge, the present study is the first to show beneficial effects of evodiamine on the pathophysiological processes of VSMCs.

Mitogenic signals, including PDGF-BB, regulate VSMC proliferation through activating diverse pathways, among which the cell cycle is a common point of convergence. Cell cycle progression is controlled by a series of protein kinases, including cyclins and CDKs (17). The activity of these cyclins and kinases are regulated by their expression levels, phosphorylation status and the presence of specific inhibitors. p21 and p27 are two negative regulators, which can arrest the cell cycle at the G0/G1 phase (18). The findings of the present study indicated that treatment with evodiamine caused cell cycle arrest in the VSMCs, accompanied with reduced expression levels of CDK 2/4/6, PCNA (a proliferation marker protein) and cyclin D1/E. By contrast, the protein levels of p27 were correspondingly increased. Although p21 has been traditionally considered to be a cyclin kinase inhibitor, it has bimodal effects on cell cycle progression and cell proliferation. The transfection of antisense p21 oligodeoxynucleotides into VSMCs leads to a decrease in the expression levels of cyclin D1 and CDK4, and results in the inhibition of PDGF-BB-induced DNA synthesis and cell proliferation (19). Therefore, the observation that PDGF-BB increased and evodiamine decreased the expression of p21 in the present study was not unusual. Of note, the inhibitory effects of evodiamine on cell cycle progression have been previously observed in various human tumor cell lines, including small-cell lung cancer cells (20), gastric cancer cells (11), breast cancer cells (21), gastric adenocarcinoma cells (22) and cervical carcinoma HeLa cells (23), and are considered to be important in the antitumor action of evodiamine. The data obtained in the present study extends the current recognition of evodiamine, and suggested that the regulation of cell cycle progression by evodiamine may be general and function in a broader range of cell types, including tumor cells and vascular cells.

MAPKs, including p38 and Erk1/2, are a family of serine/threonine kinases, which respond to various cellular stimuli, including growth factor stimulation, osmotic stress, cytokines and extracellular matrix components (24). Protein phosphorylation events are involved in the regulation of gene expression by activating transcription factors and through post-transcriptional mechanisms in MAPK-orientated signal transduction (25). In particular, mitogenesis is induced in VSMCs via the phosphorylation and activation of MAPKs, which in turn promotes VSMC proliferation under pathological conditions (26). In the present study, it was shown 
A

PCNA

CDK2

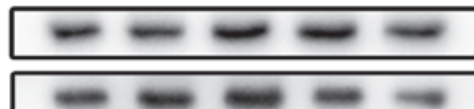

CDK4

CDK6

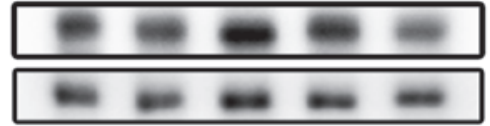

cyclin D1

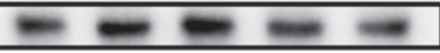

cyclin E

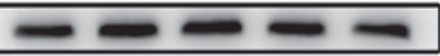

p21

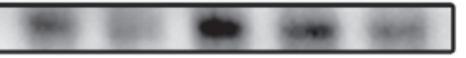

p27

GAPDH

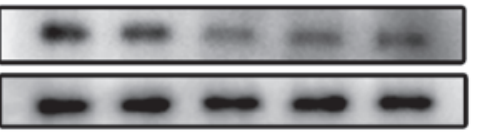

PDGF $(10 \mathrm{ng} / \mathrm{ml})$

Evo $(\mu \mathrm{M})$

0.5

$\begin{array}{ll}0.1 & 0.5\end{array}$

F

cyclin D1

G

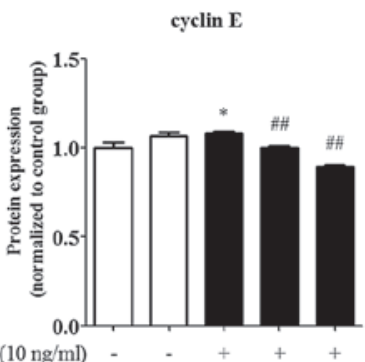

B
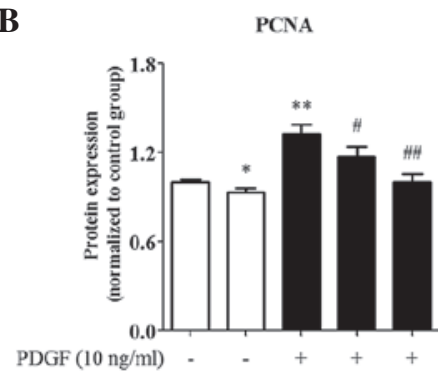

D
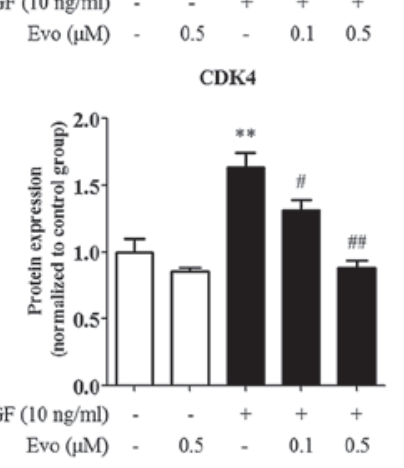

H

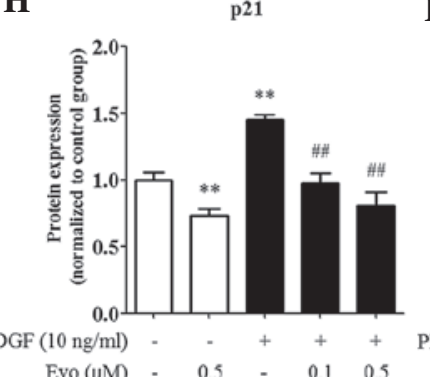

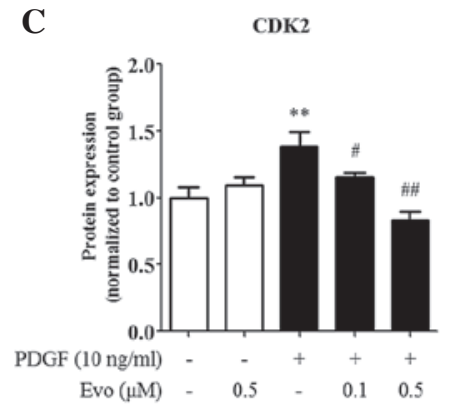

E

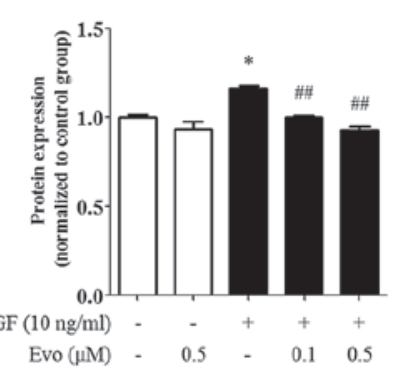

I

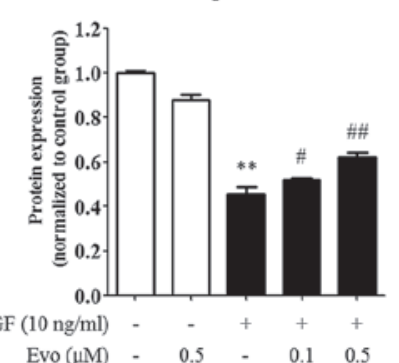

Figure 3. Evodiamine affects the protein expression levels of key regulators involved in cell cycle progression. Vascular smooth muscle cells were pretreated with $0.5 \mu \mathrm{M}$ evodiamine for $6 \mathrm{~h}$ and then stimulated with $10 \mathrm{ng} / \mathrm{ml}$ PDGF-BB for $24 \mathrm{~h}$. (A) Representative image from three separate experiments. GAPDH was used as an internal control. (B-I) Graphs of the results of statistical analyses of protein expression levels. Data are presented as the mean + standard deviation of three independent experiments. ${ }^{*} \mathrm{P}<0.05$ and ${ }^{* *} \mathrm{P}<0.01$, compared with the control group; ${ }^{~} \mathrm{P}<0.05$ and ${ }^{\# \#} \mathrm{P}<0.01$, compared with the PDGF-BB-stimulated group. Evo, evodiamine; PDGF-BB, platelet-derived growth factor-BB; PCNA, proliferating cell nuclear antigen; CDK cyclin-dependent kinase.

A

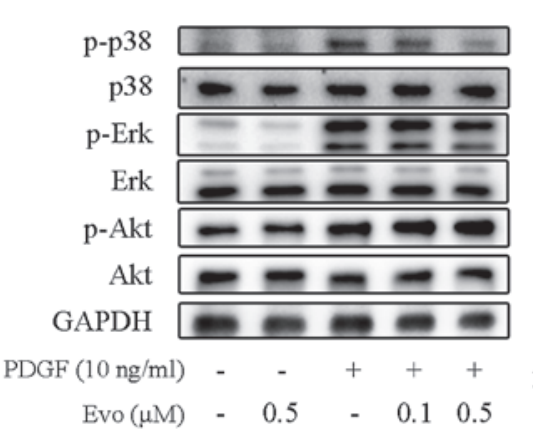

B

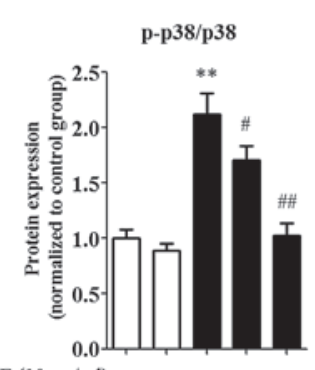

PDGF (10 ng/ml)

Evo $(\mu \mathrm{M})-0.5-0.1 \quad 0.5$
C

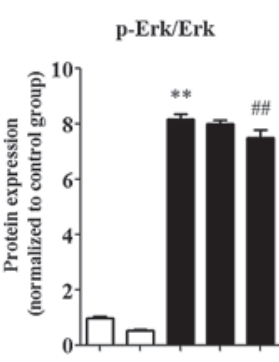

PDGF (10 ng/ml)

Evo $(\mu \mathrm{M})-0.5 \quad-0.1 \quad 0.5$
D p-Akt/akt

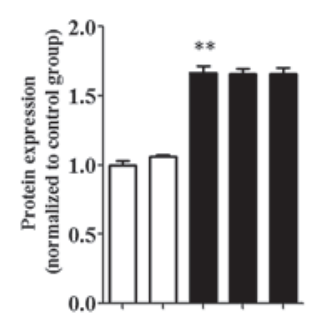

PDGF (10 ng/ml) -

Evo $(\mu \mathrm{M})-0.5-0.1-0.5$

Figure 4. Evodiamine inhibits PDGF-BB-induced kinase activation. Vascular smooth muscle cells were pretreated with 0.1 or $0.5 \mu \mathrm{M}$ evodiamine for $24 \mathrm{~h}$ and then stimulated with $10 \mathrm{ng} / \mathrm{ml}$ PDGF-BB for $15 \mathrm{~min}$. The protein expression levels of phosphorylated and total kinases were examined using qestern blot analysis. (A) Representative image from three separate experiments. GAPDH was used as an internal control. (B-D) Signal ratios of phosphorylated kinases to total kinases were calculated. Data are presented as the mean + standard deviation of three independent experiments. ${ }^{* *} \mathrm{P}<0.01$ compared with the control group; ${ }^{\#} \mathrm{P}<0.05$ and ${ }^{\# \#} \mathrm{P}<0.01$, compared with the PDGF-BB-stimulated group. Evo, evodiamine; PDGF-BB, platelet-derived growth factor-BB; ERK, extracellular signal-regulated kinase; p-, phosphorylated.

that PDGF-BB stimulation significantly increased the levels of phosphorylated p38 MAPK and Erk1/2 in VSMCs, which was inhibited by evodiamine treatment. These findings were in accordance with previous studies involving tumor cells, and suggested that the inhibition of mitogenesis and p-38/p-Erk activity may serve as the molecular basis for the functions of evodiamine $(21,27,28)$. However, the regulation of p38 and Erk activity by evodiamine is cell type-dependent and may be negative or positive. For example, whereas evodiamine inhibited the phosphorylation and activity of p38 and Erk in VSMCs 
A

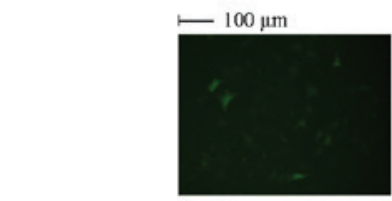

$\operatorname{PDGF}(10 \mathrm{ng} / \mathrm{ml}) \quad-$ Evo $(\mu \mathrm{M})$

C

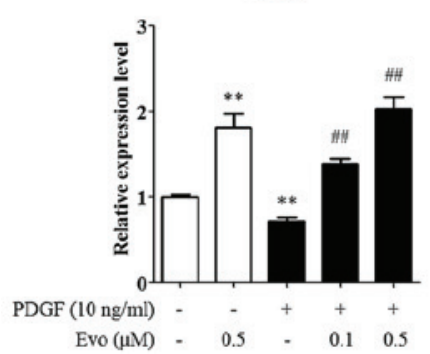

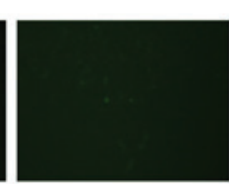

$-$

0.5

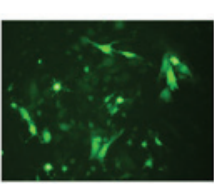

$+$

D

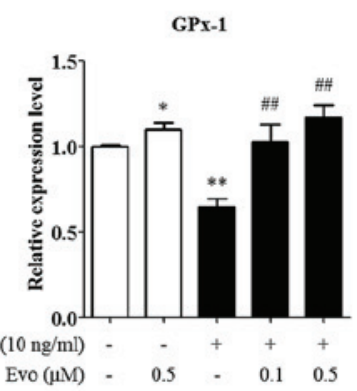

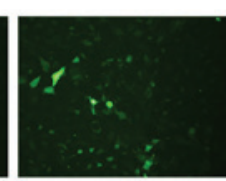

$+$
B

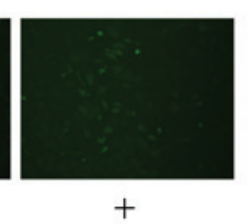

0.5

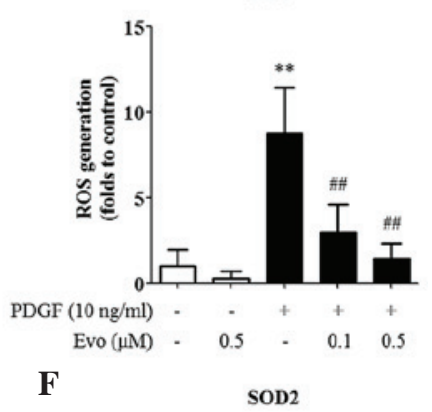

Figure 5. Evodiamine ameliorates PDGF-BB-induced oxidative stress in VSMCs. VSMCs were pretreated with 0.1 or $0.5 \mu \mathrm{M}$ evodiamine for $24 \mathrm{~h}$, and then stimulated with $10 \mathrm{ng} / \mathrm{ml}$ PDGF-BB for $1 \mathrm{~h}$. (A) ROS detection using 2' 7' dichlorofluorescin diacetate staining. Magnification, x200. (B) Quantitative data of five independent experiments, expressed as the fold increase, compared with the control. (C-F) mRNA expression levels of antioxidant genes were assessed using reverse transcription-quantitative polymerase chain reaction analysis. Data are presented as the mean+ standard deviation of three independent experiments. ${ }^{*} \mathrm{P}<0.05$ and ${ }^{* *} \mathrm{P}<0.01$, compared with the control group; ${ }^{\# \#} \mathrm{P}<0.01$, compared with the PDGF-BB-stimulated group. Evo, evodiamine; PDGF-BB, platelet-derived growth factor-BB; VSMCs, vascular smooth muscle cells; ROS, reactive oxygen species; HO-1, heme oxygenase-1; GPx-1, glutathione peroxidase 1; SOD, superoxide dismutase.

in the present study, human monocytes (14) and certain tumor cells (29), it has been reported that evodiamine activates p38 in human melanoma A375-S2 cells, stimulating the production of ROS and nitrogen oxide (30). Evodiamine also causes sustained activation of the Erk/MAPK signaling pathway in 3T3-L1 and primary preadipocytes, leading to a potent inhibitory effect for adipogenesis (31). As p38 MAPK and Erk1/2 are important intracellular signaling cascades in the regulation of several cellular activities, and are orchestrated by various upstream factors, including hormones, transcriptional factors and epigenetic regulators, it is reasonable to suggest that the regulation of p38 MAPK and Erk1/2 by evodiamine is the net output of the comprehensive actions of these factors and environmental stimuli.

The activation of phosphatidylinositol 3-kinase (PI3K) and its downstream target, Akt, is important in triggering mitogenesis (32). Although PDGF-BB increased the phosphorylation of Akt in the present study, evodiamine had a modest effect on its phosphorylation/activation. A possible explanation for this is that a difference exists in the regulation of cellular physiology by MAPKs and PI3K/Akt. For example, previous studies have indicated that Erk is critical in the regulation of cell growth, proliferation and differentiation, whereas PI3K/Akt is involved in regulating cell survival and apoptosis $(33,34)$. This was supported by a previous study showing that peroxisome proliferator-activated receptor $\delta$ agonist inhibits VSMC proliferation by significantly inhibiting the phosphorylation of Erk1/2, but not of Akt (35).

In the pathogenesis of various cardiovascular diseases, oxidative stress is critical in triggering VSMC dysfunction. Oxidative stress activates several downstream signaling molecules, including MAPKs, protein tyrosine phosphatases, protein tyrosine kinases and transcription factors (36). To protect the body from oxidative stress, endogenous antioxidant defense is evoked, which leads to the increased expression of antioxidant enzymes, including HO-1, GPx-1 and SOD. The induction of these antioxidant enzymes has anti-atherosclerotic, antidiabetic and renoprotective effects, and is critical for the maintenance of physiological homeostasis (37). In particular, these antioxidant enzymes protect VSMCs from oxidative injury and antagonize VSMC proliferation (38). The present study showed that treatment with PDGF-BB caused a more marked increase in DCFH-DA fluorescence, compared with the control VSMCs, suggesting an increased oxidative burden is induced by PDGF-BB. By contrast, evodiamine eliminated ROS generation and induced the mRNA expression levels of antioxidant enzymes in a dose-dependent manner. As increased oxidative stress is an upstream event leading to a diversity of pathophysiological changes in VSMCs, including cell cycle progression, mitogenesis and proliferation, it is essential that future investigations investigate whether the attenuation of oxidative stress by evodiamine is central or causal in its protective functions.

In conclusion, the results of the present study demonstrated that evodiamine inhibited VSMC proliferation by suppressing cell cycle progression, p38 MAPK and Erk1/2 activation, and ROS generation. These findings suggested that, in addition to its current pharmacological antitumor effects, evodiamine offers potential in the prevention and treatment of cardiovascular diseases associated with the abnormal proliferation of VSMCs.

\section{Acknowledgements}

This study was supported by grants from the National Basic Research Program of China (973 Program; 
grant nos. 2012CB947600 and 2013CB911600), the National Natural Science Foundation of China (grant nos. 31422028, 31171137, 31271261, 31401009 and 31171135), the Program for the Top Young Talents by the Organization Department of the CPC Central Committee, NSF of Jiangsu Province of China (grant no. BK20140041), the Jiangsu Planned Projects for Postdoctoral Research Funds (grant no. 1302042C), the Collaborative Innovation Center for Cardiovascular Disease Translational Medicine (Nanjing Medical University) and the Priority Academic Program Development of Jiangsu Higher Education Institutions.

\section{References}

1. Curcio A, Torella D and Indolfi C: Mechanisms of smooth muscle cell proliferation and endothelial regeneration after vascular injury and stenting: Approach to therapy. Circ J 75: 1287-1296, 2011.

2. Boucher P and Gotthardt M: LRP and PDGF signaling: A pathway to atherosclerosis. Trends Cardiovasc Med 14: 55-60, 2004.

3. Rensen SS, Doevendans PA and van Eys GJ: Regulation and characteristics of vascular smooth muscle cell phenotypic diversity. Neth Heart J 15: 100-108, 2007.

4. Park ES, Lee KP, Jung SH, Lee DY, Won KJ, Yun YP and Kim B: Compound $\mathrm{K}$, an intestinal metabolite of ginsenosides, inhibits PDGF-BB-induced VSMC proliferation and migration through G1 arrest and attenuates neointimal hyperplasia after arterial injury. Atherosclerosis 228: 53-60, 2013.

5. Palmieri C, Ravani M, Trianni G, Gianetti J, Vaghetti M, Rizza A, Paradossi U, Beqiri A and Berti S: Drug-eluting stents versus bare-metal stents in acute ST-segment elevation myocardial infarction. A single-center experience with long-term follow up. J Invasive Cardiol 22: 151-158, 2010.

6. Rosner D, McCarthy N and Bennett M: Rapamycin inhibits human in stent restenosis vascular smooth muscle cells independently of pRB phosphorylation and p53. Cardiovasc Res 66: 601-610, 2005.

7. Nguyen KT, Shaikh N, Wawro D, Zhang S, Schwade ND, Eberhart RC and Tang L: Molecular responses of vascular smooth muscle cells to paclitaxel-eluting bioresorbable stent materials. J Biomed Mater Res A 69: 513-524, 2004.

8. Zhao FH, Chen YD, Jin ZN and Lu SZ: Are impaired endothelial progenitor cells involved in the processes of late in-stent thrombosis and re-endothelialization of drug-eluting stents? Med Hypotheses 70: 512-514, 2008

9. Fei XF, Wang BX, Li TJ, Tashiro S, Minami M, Xing DJ and Ikejima T: Evodiamine, a constituent of evodiae fructus, induces anti-proliferating effects in tumor cells. Cancer Sci 94: 92-98, 2003 .

10. Jiang $\mathrm{J}$ and $\mathrm{Hu} \mathrm{C}$ : Evodiamine: A novel anti-cancer alkaloid from Evodia rutaecarpa. Molecules 14: 1852-1859, 2009.

11. Yang L, Liu X, Wu D, Zhang M, Ran G, Bi Y and Huang H: Growth inhibition and induction of apoptosis in SGC7901 human gastric cancer cells by evodiamine. Mol Med Rep 9: 1147-1152, 2014.

12. Chiou WF, Chou CJ, Shum AY and Chen CF: The vasorelaxant effect of evodiamine in rat isolated mesenteric arteries: Mode of action. Eur J Pharmacol 215: 277-283, 1992.

13. Hung PH, Lin LC, Wang GJ, Chen CF and Wang PS: Inhibitory effect of evodiamine on aldosterone release by Zona glomerulosa cells in male rats. Chin J Physiol 44: 53-57, 2001.

14. Heo SK, Yun HJ, Yi HS, Noh EK and Park SD: Evodiamine and rutaecarpine inhibit migration by light via suppression of nadph oxidase activation. J Cell Biochem 107: 123-133, 2009.

15. Gordon D, Mohai LG and Schwartz SM: Induction of polyploidy in cultures of neonatal rat aortic smooth muscle cells. Circ Res 59: 633-644, 1986.

16. Livak KJ and Schmittgen TD: Analysis of relative gene expression data using real-time quantitative PCR and the 2(-Delta Delta C(T)) Method. Methods 25: 402-408, 2001.
17. Sherr CJ: Cancer cell cycles. Science 274: 1672-1677, 1996.

18. Abukhdeir AM and Park BH: P21 and p27: Roles in carcinogenesis and drug resistance. Expert Rev Mol Med 10: e19, 2008.

19. Weiss RH, Joo A and Randour C: p21 (Waf1/Cip1) is an assembly factor required for platelet-derived growth factor-induced vascular smooth muscle cell proliferation. J Biol Chem 275: 10285-10290, 2000.

20. Fang C, Zhang J, Qi D, Fan X, Luo J, Liu L and Tan Q: Evodiamine induces $\mathrm{G} 2 / \mathrm{M}$ arrest and apoptosis via mitochondrial and endoplasmic reticulum pathways in H446 and human small-cell lung cancer cells. PLoS One 9: e115204, 2014

21. Du J, Wang XF, Zhou QM, Zhang TL, Lu YY, Zhang H and Su SB: Evodiamine induces apoptosis and inhibits metastasis in MDAMB-231 human breast cancer cells in vitro and in vivo. Oncol Rep 30: 685-694, 2013.

22. Rasul A, Yu B, Zhong L, Khan M, Yang H and Ma T: Cytotoxic effect of evodiamine in SGC-7901 human gastric adenocarcinoma cells via simultaneous induction of apoptosis and autophagy. Oncol Rep 27: 1481-1487, 2012.

23. Yang J, Wu LJ, Tashino S, Onodera S and Ikejima T: Protein tyrosine kinase pathway-derived ROS/NO productions contribute to $\mathrm{G} 2 / \mathrm{M}$ cell cycle arrest in evodiamine-treated human cervix carcinoma HeLa cells. Free Radic Res 44: 792-802, 2010.

24. Cuevas BD, Abell AN and Johnson GL: Role of mitogen-activated protein kinase kinase kinases in signal integration. Oncogene 26: 3159-3171, 2007.

25. Roux PP and Blenis J: ERK and p38 MAPK-activated protein kinases: A family of protein kinases with diverse biological functions. Microbiol Mol Biol Rev 68: 320-344, 2004.

26. Zhan Y, Kim S, Izumi Y, Izumiya Y, Nakao T, Miyazaki H and Iwao H: Role of JNK, p38 and ERK in platelet-derived growth factor-induced vascular proliferation, migration and gene expression. Arterioscler Thromb Vasc Biol 23: 795-801, 2003.

27. Yang L, Liu X, Wu D, Zhang M, Ran G, Bi Y and Huang H: Growth inhibition and induction of apoptosis in SGC7901 human gastric cancer cells by evodiamine. Mol Med Rep 9: 1147-1152, 2014.

28. Chen MC, Yu CH, Wang SW, Pu HF, Kan SF, Lin LC, Chi CW, Ho LL, Lee CH and Wang PS: Anti-proliferative effects of evodiamine on human thyroid cancer cell line ARO. J Cell Biochem 110: 1495-1503, 2010.

29. Yang J, Cai X, Lu W, Hu C, Xu X, Yu Q and Cao P: Evodiamine inhibits STAT3 signaling by inducing phosphatase shatterproof 1 in hepatocellular carcinoma cells. Cancer Lett 328: 243-251, 2013.

30. Yang J, Wu LJ, Tashiro S, Onodera S and Ikejima T: Nitric oxide activated by p38 and NF-kappaB facilitates apoptosis and cell cycle arrest under oxidative stress in evodiamine-treated human melanoma A375-S2 cells. Free Radic Res 42: 1-11, 2008.

31. Wang T, Wang Y, Kontani Y, Kobayashi Y, Sato Y, Mori N and Yamashita $\mathrm{H}$ : Evodiamine improves diet-induced obesity in a uncoupling protein-1-independent manner: Involvement of antiadipogenic mechanism and extracellularly regulated kinase/mitogen-activated protein kinase signaling. Endocrinology 149: 358-366, 2008.

32. Gao N, Zhang Z, Jiang BH and Shi X: Role of PI3K/AKT/mTOR signaling in the cell cycle progression of human prostate cancer. Biochem Biophys Res Commun 310: 1124-1132, 2003.

33. Roberts PJ and Der CJ: Targeting the Raf-MEK-ERK mitogen-activated protein kinase cascade for the treatment of cancer. Oncogene 26: 3291-3310, 2007.

34. Duronio V: The life of a cell: Apoptosis regulation by the PI3K/PKB pathway. Biochem J 415: 333-344, 2008.

35. Lim HJ, Lee S, Park JH, Lee KS, Choi HE, Chung KS, Lee HH and Park HY: PPAR delta agonist L-165041 inhibits rat vascular smooth muscle cell proliferation and migration via inhibition of cell cycle. Atherosclerosis 202: 446-454, 2009.

36. Conour JE, Graham WV and Gaskins HR: A combined in vitro/bioinformatic investigation of redox regulatory mechanisms governing cell cycle progression. Physiol Genomics 18: 196-205, 2004.

37. Pisoschi AM and Pop A: The role of antioxidants in the chemistry of oxidative stress: A review. Eur J Med Chem 97: 55-74, 2015.

38. Chen S, Ding Y, Tao W, Zhang W, Liang T and Liu C: Naringenin inhibits TNF-alpha induced VSMC proliferation and migration via induction of HO-1. Food Chem Toxicol 50: 3025-3031, 2012 